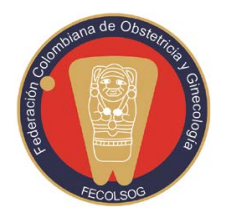

Revista Colombiana de Obstetricia y Ginecología Vol. 68 No. 1 • Enero-Marzo 2017 • (25-34)

\title{
RELACIÓN ENTRE CALIDAD DE VIDA Y PROVISIÓN DE CUIDADO PALIATIVO EN MUJERES CON CÁNCER EN COLOMBIA: UN ESTUDIO TRANSVERSAL
}

\section{Relationship between quality of life and palliative care provision in women with cancer in Colombia: A cross-sectionall study}

Ricardo Sánchez-Pedraza, MD, MSc ${ }^{1}$; Fabio Sierra-Matamoros, $\mathrm{MSc}^{2}$; Olga Lucía Morales-Mesa, MD ${ }^{3}$

Recibido: mayo 3/16 - Aceptado: marzo 2/17

\section{RESUMEN}

Objetivo: comparar los niveles de calidad de vida (CV) entre pacientes que recibieron o no cuidado paliativo $(\mathrm{CP})$, y que tenían diagnóstico de cáncer de mama u otros tipos de cáncer ginecológico.

Materiales y métodos: estudio de corte transversal efectuado en una institución de referencia en el país para diagnóstico y tratamiento del cáncer (Instituto Nacional de Cancerología de Bogotá, Colombia). En una muestra no probabilística de 114 pacientes de sexo femenino, con diagnóstico histopatológico de cáncer de mama o algún tipo de cáncer ginecológico, que aceptaran participar en el estudio, se efectuaron análisis bivariados para medir asociación entre cuidado paliativo y variables demográficas y clínicas. Se compararon puntajes de las dimensiones de calidad de vida entre grupos usando pruebas de rango con signo. Para evaluar

1 Grupo de Investigación Clínica, Instituto Nacional de Cancerología, Bogotá (Colombia). Facultad de Medicina, Universidad Nacional de Colombia, Bogotá, (Colombia).rsanchezpe@unal.edu.co

2 Instituto de Evaluación Tecnológica en Salud (IETS), Bogotá (Colombia).

3 Unidad de Cuidado Paliativo, Instituto Nacional de Cancerología, Bogotá (Colombia). asociación entre recibir cuidado paliativo y dominios de calidad de vida se utilizaron modelos de regresión logística.

Resultados: hubo 49 casos de cáncer de cérvix (42,98\%), 30 de cáncer de mama (26,32\%), 24 de ovario (21,05\%), 10 de endometrio $(8,77 \%)$ y uno de vulva $(0,88 \%)$. El $13 \%$ de las pacientes $(n=15)$ recibió CP (IC 95 \%: 8-20). Hubo mayor proporción de pacientes con estadios avanzados entre quienes recibieron $\mathrm{CP}$. Hubo menor $\mathrm{CV}$ en pacientes remitidas a CP, específicamente en los dominios bienestar físico y funcional. Los estimadores de asociación CV-CP fueron: físico (OR = 0,97; IC $95 \%$ : 0,83$1,12)$; social/familiar (OR $=1,02$; IC $95 \%$ : 0,821,27); emocional (OR = 1,10; IC $95 \%$ : 0,91-1,33); funcional (OR = 0,72; IC $95 \%$ : 0,60-0,87).

Conclusión: la frecuencia de provisión de cuidado paliativo en esta muestra es baja. Su indicación parece relacionarse más con el compromiso en la calidad de vida (específicamente en el dominio de funcionalidad) que con el estadio clínico. El cuidado paliativo es más frecuente en pacientes con mayor nivel educativo, lo cual puede relacionarse con un mayor nivel de participación en la toma de decisiones de estos pacientes. 
Palabras clave: calidad de vida, cuidados paliativos, prestación de atención de salud, neoplasias, estadificación de neoplasias, grupos de edad.

\section{ABSTRACT}

Objective: To compare quality-of-life levels (QoL) among patients who received or did not receive palliative care (PC) and had a diagnosis of breast cancer or other types of gynaecological cancers.

Materials and methods: Cross-sectional study conducted in a referral institution for the diagnosis and treatment of cancer (National Cancer Institute in Bogota, Colombia). Bivariate analyses to measure the association between PC and demographic and clinical variables were performed in a nonprobabilistic sample of 114 female patients with a hystopathological diagnosis of breast cancer or some form of gynaecological cancer who agreed to participate in the study. Scores for QoL dimensions were compared between the two groups using range tests with sign. Logistic regression models were usted to assess the association between receiving PC and QoL domains.

Results: There were 49 cases of cervical cancer (42,98\%), 30 cases $(26,32 \%)$ of breast cancer, 24 cases $(21,05 \%)$ of ovarian cancer, 10 cases $(8,77 \%)$ of endometrial cancer and 1 case $(0.88 \%)$ of vulvar cancer. Of the total number of patients, $13 \%$ $(n=15)$ received PC (95\% CI: $8 \%$ - 20\%). Among those that received PC there was a higher proportion of patients with advanced stages. QoL scores were lower in patients referred to PC, especially for the physical wellness and functional domains. The estimators for QoL/PC association were: physical $(\mathrm{OR}=0.97 ; 95 \% \mathrm{CI} 0.83-1.12) ;$ social/family (OR $=1.02 ; 95 \%$ CI 0.82-1.27); emotional $(\mathrm{OR}=1.10$; $95 \%$ CI 0.91-1.33); functional (OR $=0.72 ; 95 \%$ CI 0.60-0.87).

Conclusion: The frequency with which PC was provided in this sample is low. The indication for PC appears to be related more to QoL compromise (specifically in the functionality domain) than to the clinical stage of the disease. PC is more frequent among patients with a higher level of education, which may be related to their greater participation in decision-making.

Key words: Quality of life, palliative care, healthcare provision, neoplasms, staging of neoplasms, age groups.

\section{INTRODUCCIÓN}

Una de las metas del cuidado del paciente con cáncer es optimizar su calidad de vida, lo cual implica intervenir en múltiples dimensiones además del control de síntomas, como son las relacionadas con los aspectos psicológicos, funcionales, sociales y espirituales (1).

La importancia de la calidad de vida relacionada con la salud se ha reflejado en su incorporación como un desenlace central, no solo en los estudios en pacientes con cáncer $(2,3)$, sino también en otros escenarios clínicos (4). Sin embargo, su medición es complicada por el gran componente de subjetividad implícito en este concepto (5); por ejemplo, se ha reportado que personas que a juicio de evaluadores externos tendrían muy buena calidad de vida reportan todo lo contrario, mientras que pacientes con discapacidades graves y persistentes refieren muy buenos niveles de calidad de vida, fenómeno conocido como la paradoja de discapacidad (6). Por otra parte, se ha descrito que factores transculturales pueden influir de manera importante en la calificación que le dan las personas a esta dimensión $(7,8)$, que aspectos tales como el acceso a sistemas de salud influye positivamente sobre la calidad de vida (9), o que las dimensiones de religiosidad y espiritualidad pueden influir en la calificación que una persona le dé a su calidad de vida relacionada con la salud $(10,11)$.

En este contexto, se han desarrollado herramientas para intentar medir este concepto subjetivo de manera universal, válida y confiable (12); las que más ampliamente se han utilizado en pacientes con cáncer son las escalas de la European Organization for Research and Treatment of Cancer (EORTC) (13), y la de Functional Assessment of Chronic Ill- 
ness Therapy (FACIT) $(14,15)$, aunque también se han propuesto instrumentos que le den más énfasis al componente subjetivo del constructo (16-18). Independientemente del uso de instrumentos objetivos y validados, la interpretación de los resultados de las mediciones con estas escalas también puede verse afectada por el tipo de análisis estadístico utilizado (19).

Para la Organización Mundial de la Salud (OMS), la intervención que tiene en cuenta como foco central la calidad de vida del paciente es el cuidado paliativo (CP), y lo define como "un enfoque que mejora la calidad de vida de los pacientes y sus familias, enfrentando los problemas asociados con la enfermedad que amenaza la vida, a través de la prevención y el alivio del sufrimiento por medio de la identificación temprana y el manejo impecable del dolor y otros problemas físicos, psicosociales y espirituales" (20). Esta repercusión favorable del CP sobre la calidad de vida se ha reportado en diferentes estudios (21-24) y también en desenlaces clínicos diferentes a la calidad de vida (aunque relacionados) como la satisfacción del paciente con el cuidado de su salud, la supervivencia, la mejoría de síntomas físicos y emocionales, menores dificultades en la interacción entre el paciente y los profesionales de la salud y la reducción en la utilización de recursos del sistema de salud (25-28). También se ha planteado que la provisión temprana de cuidado paliativo especializado, además de mejorar la calidad de vida, disminuye los gastos en salud y ayuda a clarificar las preferencias del tratamiento y las metas de cuidado en pacientes con cáncer en estadios avanzados (29). Adicionalmente, se ha propuesto que las intervenciones tempranas en $\mathrm{CP}$ no solo mejoran la calidad de vida, sino que ayudan a un mejor control de síntomas, reducen las dificultades en la interacción entre clínicos y pacientes y mejoran la satisfacción tanto del paciente como de sus cuidadores $(30,31)$.

Existen datos provenientes de ensayos clínicos que han recomendado que el CP se ofrezca al paciente cuando se le haga el diagnóstico inicial de cáncer, teniendo en cuenta que esta intervención temprana puede tener efectos benéficos sobre diferentes desenlaces clínicos, especialmente la calidad de vida (32). A pesar de esto, esta intervención es generalmente vista como una prioridad menor cuando se establecen los blancos de las terapias en cáncer, ya que se maneja una concepción de CP más asociada con el cuidado del moribundo (33). Se ha observado que la referencia a este tipo de tratamiento se hace de manera tardía, en promedio unos dos meses antes de la muerte del paciente $(34,35)$.

Es poca la información disponible a nivel local sobre la prevalencia del uso del CP en pacientes con cáncer, como también sobre cuáles son dominios del constructo de la calidad de vida que se asocian con recibir este cuidado. Por otra parte, se tiene la percepción de que a pesar de existir un compromiso en la calidad de vida del paciente este no es remitido a $\mathrm{CP}$, y que tener información sobre la calidad de vida de las pacientes podría orientar al equipo de salud sobre la necesidad de iniciar tempranamente el CP.

Teniendo en cuenta los antecedentes expuestos, se plantearon como objetivos del presente estudio evaluar la prevalencia de utilización de $\mathrm{CP}$ en pacientes con diagnóstico de cáncer de mama y otros tipos de cáncer ginecológico teniendo en cuenta los diferentes estadios de la enfermedad, y comparar los niveles de calidad de vida entre pacientes que recibieron o no $\mathrm{CP}$.

\section{MATERIALES Y MÉTODOS}

Estudio transversal efectuado en pacientes con diagnóstico de cáncer ginecológico o de mama, histopatológicamente confirmado y en cualquier estadio clínico, que acudieron al Instituto Nacional de Cancerología (INC), una institución de referencia para diagnóstico y tratamiento del cáncer en Bogotá, Colombia, entre el 2011 y el 2013. A esta institución acuden principalmente pacientes del régimen subsidiado por el Estado en sistema de seguridad social en Colombia. Para el estudio se recurrió a una muestra no probabilística, consecutiva.

Las pacientes fueron ubicadas por medio del sistema de registro interno del INC. Una vez ve- 
rificado el diagnóstico histopatológico, y después de obtener la firma del consentimiento informado, un auxiliar de investigación registró variables demográficas y clínicas y aplicó la escala FACT-G, validada en Colombia (36), para medir la calidad de vida. Este instrumento está conformado por 27 ítems, cada uno de los cuales es calificado con una escala ordinal con cinco categorías que van desde cero hasta cuatro; los 27 ítems conforman cuatro dimensiones de bienestar (físico, social y familiar, emocional y de funcionamiento). Los mayores puntajes corresponden a mejores niveles de calidad de vida. Cada dominio tiene un puntaje entre 0 y 28, con excepción del dominio relacionado con aspectos emocionales (que tiene un puntaje entre 0 y 24). El auxiliar de investigación que efectuó las mediciones recibió entrenamiento para asistir a los pacientes cuando estuvieran contestando la escala FACIT-G. La calificación del instrumento se efectuó utilizando los algoritmos recomendados por la organización FACIT (15). La provisión de cuidado paliativo fue definida como la remisión e inicio de la atención en el servicio de cuidados paliativos del INC. Las pacientes que recibieron cuidados paliativos fueron evaluadas máximo una semana después de iniciada esa intervención.

Las variables demográficas contempladas en el estudio fueron edad, estrato socioeconómico, procedencia (Bogotá sí o no) y nivel de escolaridad. Como variables clínicas se consideraron la localización del cáncer (mama u otros tipos de cáncer ginecológico), la estadificación y la provisión de cuidados paliativos.

El cálculo del tamaño de muestra se efectuó con base en la hipótesis nula de que la provisión de CP depende del compromiso en los diferentes dominios del constructo de calidad de vida. Se tuvo en cuenta la posibilidad de encontrar diferencias mínimas importantes de al menos tres puntos en cada dominio de la escala (14); con tales diferencias, dados valores de desviación estándar de cinco en cada grupo (con y sin CP), una significación del 5 \% y un poder del $80 \%$, se requieren al menos 45 pacientes en cada uno de los grupos (cálculos efectuados con el programa PASS(R). Para el componente descriptivo del análisis estadístico se manejaron las herramientas convencionales (medias o medianas con sus medidas de dispersión, o proporciones dependiendo del tipo de variable). La frecuencia de utilización de CP fue calculada como porcentaje y se reportaron sus intervalos de confianza del $95 \%$ asumiendo una distribución binomial. Se efectuaron análisis bivariados para evaluar la asociación entre $\mathrm{CP}$ y cada una de las diferentes variables demográficas y clínicas incorporadas en el estudio; para dichos análisis se utilizaron pruebas $t$ o pruebas exactas de Fisher. La utilización de pruebas $t$ para muestras independientes se basó en la evaluación de simetría de las variables mediante la inspección de gráficos de cajas y los resultados de los valores de sesgo. La comparación de los puntajes de las diferentes dimensiones de calidad de vida entre pacientes que recibieron o no cuidado paliativo se realizó con pruebas de suma de rangos. Para evaluar eventuales patrones de asociación entre recibir $\mathrm{CP}$ y dominios de calidad de vida se utilizó un modelo de regresión logística tomando como variable dependiente el recibir o no $\mathrm{CP}$, y como variable independiente los diferentes dominios en la escala de calidad de vida; en dicho modelo se incorporaron como covariables aquellas variables demográficas o clínicas que en el análisis bivariado preliminar mostraron asociación con la variable "cuidado paliativo" utilizando un criterio de significación laxo ( $p<0,2)$. Para todos los demás casos que implicaron pruebas de hipótesis se manejaron niveles de significación del $5 \%$ e hipótesis a dos colas. La fase inicial de construcción del modelo incluyó como covariables la edad, localización del cáncer, años de escolaridad, estrato socioeconómico, procedencia y estadio clínico. Debido a la baja frecuencia de pacientes con estadio IV, para el modelo logístico se recategorizó esta variable dejándose solo dos niveles (estadio $<3$ o $>=3$ : esta última se tomó como categoría de referencia). Se incluyeron términos de interacción con estos niveles de categorización de la variable. 
Para el modelo multivariable los datos faltantes fueron manejados con borrado de lista. El análisis estadístico se efectuó con el programa R.

La recolección de los datos del presente estudio se enmarca dentro de la realización de un proyecto para evaluar calidad de vida en pacientes con cáncer, aprobado por el comité de ética del Instituto Nacional de Cancerología.

\section{RESULTADOS}

De las 114 pacientes que ingresaron en el estudio, $30(26,32 \%)$ presentaron cáncer de mama y el resto algún otro tipo de cáncer ginecológico: 49 casos de cáncer de cérvix (42,98\%), 24 de ovario (21,05\%), 10 de endometrio (8,77\%) y uno de vulva $(0,88 \%)$. El $13 \%$ de las pacientes $(\mathrm{N}=15)$ recibió CP (IC $95 \%$ : 8-20\%). El CP se brindó al $15 \%$ de las pacientes con cáncer ginecológico $(\mathrm{N}=84$; IC $95 \%$ : 8-23) y al $7 \%$ de las pacientes con cáncer de mama (IC $95 \%$ : 0-16). Dependiendo del estadio clínico la frecuencia de provisión de $\mathrm{CP}$ fue la siguiente: Estadio 1: 3,3\% ( $=1$; IC $95 \%$ : 0-10); estadio 2: $12 \%(\mathrm{~N}=25$; IC $95 \%$ : 0-26); estadio 3: $18 \%(\mathrm{~N}=40$; IC $95 \%$ : 5-30); estadio 4: 50\% $(\mathrm{N}=8$; IC $95 \%$ : 5-95).

La edad tuvo una media de 55,5 años (DE $\pm 12,8$ años): en el grupo de pacientes sin CP la media de edad fue de 55,8 años (DE $\pm 12,7$ años) y en el grupo con CP fue de 53,5 años ( $\mathrm{DE}=13,9$ años); la diferencia en las medias entre estos dos grupos no fue significativa. La escolaridad tuvo una media de 7,5 años (DE $\pm 7,5$ ), siendo de 7,04 años (DE \pm 5,2 años) en quienes no recibieron cuidados paliativos y de 10,5 (DE $\pm 4,1$ años) en el grupo con este tipo de tratamiento; esta diferencia de medias fue significativa $\left(\mathrm{t}_{(112)}=-2,4 ; \mathrm{p}=0,02\right)$.

Las características demográficas y clínicas medidas en este estudio se muestran en la tabla 1; puede verse que existe una diferencia significativa en las proporciones de pacientes que reciben $\mathrm{CP}$ según el estadio clínico (hay mayor proporción de pacientes con estadios clínicos avanzados entre quienes reciben $\mathrm{CP}$ ).
En relación con los cuatro dominios del constructo de calidad de vida se encontró que la calidad de vida es menor en los pacientes remitidos a $\mathrm{CP}$, lo cual ocurre a expensas de los dominios de bienestar físico (Prueba de suma de rangos, $\mathrm{z}=2,24$, $\mathrm{p}=0,042$ ) y funcional (Prueba de suma de rangos, $\mathrm{z}=3,7, \mathrm{p}=0,0002)($ tabla 2).

La asociación entre los dominios de calidad de vida y recibir $\mathrm{CP}$ se evaluó mediante estimación de OR utilizando un modelo logístico. Los valores de estos estimadores fueron: para bienestar físico $(\mathrm{OR}=0,92$; IC $95 \%$ : 0,8-1,1); para bienestar social y familiar (OR = 1,07; IC $95 \%: 0,9-1,3)$; bienestar emocional (OR = 1,1; IC $95 \%$ 0,94-1,4), y bienestar funcional $(\mathrm{OR}=0,7$; IC $95 \%$ : 0,6-0,9); esto indica que la provisión de $\mathrm{CP}$ es más frecuente entre menores sean los niveles de calidad de vida relacionada con el dominio de funcionalidad. $\mathrm{Al}$ incorporar en el modelo multivariable las variables de control que mostraron aceptables niveles de significación no se encontró un cambio clínicamente significativo en la magnitud de los estimadores (tabla 3).

\section{DISCUSIÓN}

En esta muestra de pacientes con cáncer de mama o cáncer ginecológico se encontró un porcentaje bajo de pacientes con CP. Esta proporción es baja en un centro de referencia nacional, si se tienen en cuenta las recomendaciones de que, independientemente del estadio clínico, los pacientes deberían recibir tempranamente atención de CP (32). Considerando este hallazgo, sería importante efectuar estudios similares en otros centros de atención con características parecidas a las del Instituto Nacional de Cancerología de Bogotá, o explorar formalmente cuáles son los criterios que se utilizan en la práctica clínica para realizar la remisión de pacientes a CP. Estudios prospectivos o encuestas a médicos podrían dar información adicional sobre los patrones de remisión a CP. En estudios que también reportan baja frecuencia de remisión de pacientes a $\mathrm{CP}$ se ha recomendado el establecimiento de clínicas ambulatorias de estos cuidados y la educación en CP a los oncólogos (37). 


\begin{tabular}{|c|c|c|c|c|c|}
\hline & & \multicolumn{2}{|c|}{ Sin cuidado paliativo } & \multicolumn{2}{|c|}{ Con cuidado paliativo } \\
\hline & & $\mathbf{n}$ & $\%$ & $\mathbf{n}$ & $\%$ \\
\hline \multirow{6}{*}{$\begin{array}{l}\text { Estrato } \\
\text { socioeconómico }^{1}\end{array}$} & 1 & 16 & 16,16 & 2 & 13,33 \\
\hline & 2 & 41 & 42,42 & 3 & 20 \\
\hline & 3 & 32 & 33,33 & 9 & 60 \\
\hline & 4 & 2 & 2,02 & 1 & 6,67 \\
\hline & 5 & 1 & 1,01 & 0 & 0 \\
\hline & 6 & 5 & 5,05 & 0 & 0 \\
\hline \multirow{2}{*}{ Procedencia $^{2}$} & Fuera de Bogotá & 44 & 44,44 & 4 & 26,67 \\
\hline & Bogotá & 55 & 55,56 & 11 & 73,33 \\
\hline \multirow{2}{*}{$\begin{array}{l}\text { Localización del } \\
\text { cáncer }^{3}\end{array}$} & Ginecológicos & 71 & 71,72 & 13 & 86,67 \\
\hline & Mama & 28 & 28,28 & 2 & 13,33 \\
\hline \multirow{4}{*}{ Estadio clínico ${ }^{4}$} & 1 & 29 & 32,95 & 1 & 6,67 \\
\hline & 2 & 22 & 25,00 & 3 & 20 \\
\hline & 3 & 33 & 37,50 & 7 & 46,67 \\
\hline & 4 & 4 & 4,55 & 4 & 26,67 \\
\hline
\end{tabular}

${ }^{1}$ Prueba exacta de Fisher: $\mathrm{p}=0,24$

${ }^{2}$ Prueba exacta de Fisher: $\mathrm{p}=0,27$

${ }^{3}$ Prueba exacta de Fisher: $\mathrm{p}=0,35$

${ }^{4}$ Prueba exacta de Fisher: $\mathrm{p}=0,014$

\section{Tabla 2.}

\section{Niveles de calidad de vida según provisión de cuidado paliativo}

\begin{tabular}{|l|c|c|c|}
\hline \multirow{2}{*}{ Dominio FACIT-G } & \multicolumn{3}{|c|}{ Media (DE) } \\
\cline { 2 - 4 } & Total muestra & Sin cuidado paliativo & Con cuidado paliativo \\
\hline Bienestar físico $^{1}$ & $20,1( \pm 5,9)$ & $20,6( \pm 5,6)$ & $16,8( \pm 6,6)$ \\
\hline Bienestar social y familiar $^{2}$ & $18,5( \pm 4,3)$ & $18,56( \pm 4,38)$ & $17,9( \pm 4,1)$ \\
\hline Bienestar emocional $^{3}$ & $16,4( \pm 4,6)$ & $16,5( \pm 4,6)$ & $15,5( \pm 4,2)$ \\
\hline Bienestar funcional $^{4}$ & $16,5( \pm 5,1)$ & $17,4( \pm 4,4)$ & $10,9( \pm 5,9)$ \\
\hline Puntaje total $^{5}$ & $17,9( \pm 5,2)$ & $73,1( \pm 13,5)$ & $60,9( \pm 15,0)$ \\
\hline
\end{tabular}

${ }^{1}$ Prueba de suma de rangos, $\mathrm{z}=2,24, \mathrm{p}=0,042$

${ }^{2}$ Prueba de suma de rangos, $\mathrm{z}=0,51, \mathrm{p}=0,611$

${ }^{3}$ Prueba de suma de rangos, $\mathrm{z}=0,97, \mathrm{p}=0,332$

${ }^{4}$ Prueba de suma de rangos, $\mathrm{z}=3,71, \mathrm{p}=0,0002$

${ }^{5}$ Prueba de suma de rangos, $\mathrm{z}=2,92, \mathrm{p}=0,004$ 


\begin{tabular}{|l|c|c|}
\hline \multicolumn{2}{|c|}{$\begin{array}{c}\text { Tabla } 3 . \\
\text { Modelo multivariado ajustado para evaluar asociación entre cuidado paliativo } \\
\text { y dominios de calidad de vida* }\end{array}$} \\
\hline & OR & IC 95\% \\
\hline Bienestar físico & 0,97 & $0,83-1,12$ \\
\hline Bienestar social y familiar & 1,02 & $0,82-1,27$ \\
\hline Bienestar emocional & 1,10 & $0,91-1,33$ \\
\hline Bienestar funcional & 0,71 & $0,60-0,86$ \\
\hline Años de escolaridad & 1,26 & $1,06-1,5$ \\
\hline Estadio & 3,03 & $0,57-16,1$ \\
\hline
\end{tabular}

* En la tabla solo se reportan las covariables que en el análisis bivariado mostraron valores $\mathrm{p}>0,2$.

Otro hallazgo importante del estudio es que los pacientes que presentan menores niveles de calidad de vida son los remitidos con más frecuencia a atención por CP. Estos niveles bajos de calidad de vida reflejan principalmente los dominios de funcionalidad y bienestar físico, y parecen ser independientes del estadio clínico. Este hallazgo es consistente con reportes previos de estudios efectuados en pacientes con cáncer que sugieren independencia entre calidad de vida y estadio clínico (38). Los hallazgos del presente estudio sugieren que las dimensiones relacionadas con lo social-familiar y con lo afectivo parecen no ser tenidas en cuenta para que los médicos tratantes decidan una intervención más integral para sus pacientes; esta posible falta de atención a los aspectos psicosociales del paciente podría generar demoras en el manejo de aspectos importantes en su tratamiento (se ha reportado que hasta un 20\% de pacientes en $\mathrm{CP}$ pueden presentar trastornos depresivos mayores que requieren tratamiento) (39).

Aunque en el análisis bivariado el estadio clínico mostró asociación con el tratamiento en CP (es más frecuente el tratamiento de $\mathrm{CP}$ en pacientes con estadios avanzados), esta asociación pierde significación estadística si se tiene en cuenta el efecto de los niveles de calidad de vida. Esto sugiere que la remisión a $\mathrm{CP}$ se hace más teniendo en cuenta el compromiso en la calidad de vida, especialmente en lo relacionado con funcionalidad, independientemente del estadio clínico; de esta manera, pacientes con estadios clínicos avanzados pero buenos niveles de funcionalidad tendrían menos remisión a CP que pacientes con pobres niveles de funcionalidad, independientemente del estadio clínico.

Otro hallazgo que se destaca en el presente estudio es la asociación entre años de escolaridad y CP; este ha sido reportado previamente en otros estudios y se ha relacionado con un mayor nivel de empoderamiento, autodeterminación, participación en la toma de decisiones y control sobre el final de la vida en pacientes con buenos niveles educativos (40, 41). Lo anterior sugiere que en pacientes con bajo nivel educativo deberían plantearse intervenciones que atenúen esta situación desventajosa y que mejoren su posibilidad de acceder al tratamiento de $\mathrm{CP}$.

Como limitaciones del presente estudio se reconocen varios aspectos: 1) la baja representación en la muestra de pacientes con estratos socioeconómicos altos puede relacionarse con algún sesgo de selección y afectar la generalización de los resultados; 2) pueden existir otras variables relacionadas con la provisión de $\mathrm{CP}$, como pueden ser las características de acceso dentro del sistema de salud, que no fueron consideradas dentro del presente estudio; 3) la baja frecuencia de pacientes con CP pudo haber comprometido el poder del estudio para encontrar 
diferencias entre grupos; 4) los posibles sesgos de selección derivados de un muestreo no probabilístico, y los sesgos relacionados con haber incorporado en un solo conglomerado diferentes tipos de cáncer.

\section{CONCLUSIONES}

La frecuencia de provisión de CP en esta muestra es baja. La indicación de CP parece relacionarse más con compromiso en la calidad de vida (específicamente en el dominio de funcionalidad) que con estadio clínico. El CP es más frecuente entre pacientes con nivel educativo más alto, lo cual puede relacionarse con el mayor nivel de participación en la toma de decisiones de estos pacientes.

\section{FINANCIACIÓN}

El estudio fue financiado por el Instituto Nacional de Cancerología y la Universidad Nacional de Colombia.

\section{REFERENCIAS}

1. Jocham HR, Dassen T, Widdershoven G, Halfens R. Quality of life in palliative care cancer patients: A literature review. J Clin Nurs. 2006;15:1188-95.

2. Kiebert GM, Curran D, Aaronson NK. Quality of life as an endpoint in EORTC clinical trials. European Organization for Research and Treatment for Cancer. Stat Med. 1998;17:561-9.

3. Aaronson NK. Assessing the quality of life of patients in cancer clinical trials: Common problems and common sense solutions. Eur J Cancer. 1992;28A:1304-7.

4. Higginson IJ, Carr AJ. Measuring quality of life: Using quality of life measures in the clinical setting. BMJ. 2001;322:1297-300

5. Slevin ML, Plant H, Lynch D, Drinkwater J, Gregory WM. Who should measure quality of life, the doctor or the patient? British Journal of Cancer. 1988;57:109-12.

6. Albrecht GL, Devlieger PJ. The disability paradox: High quality of life against all odds. Soc Sci Med. 1999;48:977-88.

7. Camfield L, Skevington SM. On subjective well-being and quality of life. Journal of Health Psychology. 2008;13:764-75
8. Molzahn AE, Kalfoss M, Schick Makaroff K, Skevington SM. Comparing the importance of different aspects of quality of life to older adults across diverse cultures. Age and Ageing. 2011;40:192-9.

9. Sánchez-Pedraza R, Ballesteros MP, Anzola JD. The concept of quality of life in women suffering from cancer. Rev Salud Pública (Bogotá). 2010;12:889-902.

10. O’Connell KA, Skevington SM. Spiritual, religious, and personal beliefs are important and distinctive to assessing quality of life in health: A comparison of theoretical models. British Journal of health Psychology. 2010;15(Pt 4):729-48

11. O'Connell KA, Skevington SM. To measure or not to measure? Reviewing the assessment of spirituality and religion in health-related quality of life. Chronic illness. 2007;3:77-87.

12. Bowling A. Measuring health: a review of quality of life measurement scales. (3rd ed). Maidenhead, Berkshire, England; New York: Open University Press; 2005. xii. p. 211.

13. Aaronson NK, Ahmedzai S, Bergman B, Bullinger M, Cull A, Duez NJ, et al. The European Organization for Research and Treatment of Cancer QLQ-C30: A quality-of-life instrument for use in international clinical trials in oncology. Journal of the National Cancer Institute. 1993;85:365-76.

14. Webster K, Cella D, Yost K. The Functional Assessment of Chronic Illness Therapy (FACIT) Measurement System: Properties, applications, and interpretation. Health Qual Life Outcomes. 2003;1:79.

15. Cella DF, Tulsky DS, Gray G, Sarafian B, Linn E, Bonomi A, et al. The Functional Assessment of Cancer Therapy scale: Development and validation of the general measure. Journal of Clinical Oncology. 1993;11:570-9.

16. Joyce CR, Hickey A, McGee HM, O’Boyle CA. A theory-based method for the evaluation of individual quality of life: the SEIQoL. Quality of life research: An international journal of quality of life aspects of treatment, care and rehabilitation. 2003;12(3):275-80.

17. Macduff C. Respondent-generated quality of life measures: Useful tools for nursing or more fool's gold? J Adv Nurs. 2000;32:375-82. 
18. Ruta DA, Garratt AM, Leng M, Russell IT, MacDonald LM. A new approach to the measurement of quality of life. The Patient-Generated Index. Medical Care. 1994;32:1109-26.

19. Curran D, Aaronson N, Standaert B, Molenberghs G, Therasse P, Ramirez A, et al. Summary measures and statistics in the analysis of quality of life data: An example from an EORTC-NCIC-SAKK locally advanced breast cancer study. Eur J Cancer. 2000;36:834-44.

20. Organization WH. Definition of Palliative Care 2012 [visitado 2016 Feb 28]. Disponible en: http://www. who.int/cancer/palliative/definition/en

21. El-Jawahri A, Greer JA, Temel JS. Does palliative care improve outcomes for patients with incurable illness? A review of the evidence. The Journal of Supportive Oncology. 2011;9:87-94.

22. Lorenz KA, Lynn J, Dy SM, Shugarman LR, Wilkinson A, Mularski RA, et al. Evidence for improving palliative care at the end of life: A systematic review. Annals of Internal Medicine. 2008;148:147-59.

23. Zimmermann C, Riechelmann R, Krzyzanowska M, Rodin G, Tannock I. Effectiveness of specialized palliative care: A systematic review. Jama. 2008;299:1698-709.

24. Rabow M, Kvale E, Barbour L, Cassel JB, Cohen S, Jackson V, et al. Moving upstream: A review of the evidence of the impact of outpatient palliative care. Journal of Palliative Medicine. 2013;16:1540-9.

25. Casarett D, Pickard A, Bailey FA, Ritchie C, Furman C, Rosenfeld K, et al. Do palliative consultations improve patient outcomes? Journal of the American Geriatrics Society. 2008;56:593-9.

26. Higginson IJ, Evans CJ. What is the evidence that palliative care teams improve outcomes for cancer patients and their families? Cancer Journal. 2010;16:423-35.

27. Follwell M, Burman D, Le LW, Wakimoto K, Seccareccia D, Bryson J, et al. Phase II study of an outpatient palliative care intervention in patients with metastatic cancer. Journal of Clinical Oncology. 2009;27:206-13.

28. Rabow MW, Dibble SL, Pantilat SZ, McPhee SJ. The comprehensive care team: A controlled trial of outpatient palliative medicine consultation. Archives of internal Medicine. 2004;164:83-91.

29. Parikh RB, Kirch RA, Smith TJ, Temel JS. Early specialty palliative care--translating data in oncology into practice. The New England Journal of Medicine. 2013;369:2347-51.

30. McDonald J, Swami N, Hannon B, Lo C, Pope A, Oza A, et al. Impact of early palliative care on caregivers of patients with advanced cancer: Cluster randomised trial. Ann Oncol. 2016. pii: mdw438. doi: 10.1093/ annonc/mdw438

31. Zimmermann C, Swami N, Krzyzanowska M, Hannon B, Leighl N, Oza A, et al. Early palliative care for patients with advanced cancer: a cluster-randomised controlled trial. Lancet. 2014;383:1721-30.

32. Smith TJ, Temin S, Alesi ER, Abernethy AP, Balboni TA, Basch EM, et al. American Society of Clinical Oncology provisional clinical opinion: the integration of palliative care into standard oncology care. Journal of Clinical Oncology. 2012;30:880-7.

33. Prioritising palliative care. Lancet. 2014;383:1694. doi: 10.1016/S0140-6736(14)60814-X.

34. Lamont EB, Christakis NA. Physician factors in the timing of cancer patient referral to hospice palliative care. Cancer. 2002;94:2733-7.

35. Wentlandt K, Krzyzanowska MK, Swami N, Rodin GM, Le LW, Zimmermann C. Referral practices of oncologists to specialized palliative care. Journal of Clinical Oncology. 2012;30:4380-6.

36. Sánchez R, Ballesteros M, Arnold BJ. Validation of the FACT-G scale for evaluating quality of life in cancer patients in Colombia. Quality of life research: An international journal of quality of life aspects of treatment, care and rehabilitation. 2011;20:19-29.

37. Hannon B, Swami N, Pope A, Leighl N, Rodin G, Krzyzanowska M, et al. Early palliative care and its role in oncology: A qualitative study. Oncologist. 2016.

38. Sanchez R, Alexander-Sierra F, Oliveros R. Relationship between quality of life and clinical status in patients with gastrointestinal cancer. Rev Esp Enferm Dig. 2012;104:584-91. 
39. Rayner L, Lee W, Price A, Monroe B, Sykes N, Hansford P, et al. The clinical epidemiology of depression in palliative care and the predictive value of somatic symptoms: cross-sectional survey with four-week follow-up. Palliat Med. 2011;25:229-41.

40. Bossuyt N, van den Block L, Cohen J, Meeussen K, Bilsen J, Echteld M, et al. Is individual educational level related to end-of-life care use? Results from a nationwide retrospective cohort study in Belgium. Journal of Palliative Medicine. 2011;14:1135-41.

41. Chambaere K, Rietjens JA, Cohen J, Pardon K, Deschepper R, Pasman HR, et al. Is educational attainment related to end-of-life decision-making? A large post-mortem survey in Belgium. BMC Public Health. 2013;13:1055. 